\title{
Robot-assisted radical cystectomy: patient selection and special considerations
}

This article was published in the following Dove Press journal:

Robotic Surgery: Research and Reviews

19 October 2017

Number of times this article has been viewed

\author{
Mevlana Derya Balbay' \\ Erdem Koc ${ }^{2}$ \\ Abdullah Erdem Canda ${ }^{3}$ \\ 'Department of Urology, American \\ Hospital, Istanbul, Turkey; \\ ${ }^{2}$ Department of Urology, Ankara \\ Ataturk Training and Research \\ Hospital, Ankara, Turkey; ${ }^{3}$ Department \\ of Urology, School of Medicine, \\ Ankara Ataturk Training and Research \\ Hospital,Yildirim Beyazit University, \\ Ankara, Turkey
}

\begin{abstract}
Robot-assisted (RA) procedures are increasingly being performed as minimally invasive surgical approaches. Less insensible losses due to a closed abdomen, smaller incisions with less retractor strain, decreased analgesic requirements, and earlier postoperative ambulation are suggested advantages of robot-assisted radical cystectomy (RARC). Patients who undergo open radical cystectomy are also candidates for RARC procedure. However, the steep Trendelenburg position and pneumoperitoneum develop a non-physiological condition. Intraabdominal adhesions preventing the placement of the ports and patients who cannot tolerate the pneumoperitoneum and/or steep Trendelenburg position are special contraindications of RARC. Besides, body mass index $>30 \mathrm{~kg} / \mathrm{m}^{2}$, presence of extravesical disease, bulky lymphadenopathy, previous vascular surgery, previous distal colorectal surgery, previous pelvic radiation, previous pelvic trauma, and/or preexisting cardiovascular/pulmonary disease that is compromised with positioning are not certainly contraindicated but unwanted conditions in which the RARC may be performed successfully as the surgeons gain experience.
\end{abstract}

Keywords: robotic cystectomy, patient selection, intracorporeal urinary diversion

\section{Introduction}

Open radical cystectomy (ORC) with extended pelvic lymph node dissection (PLND) is the "gold standard" surgical approach in patients with muscle-invasive bladder cancer, in addition to patients with high-grade, recurrent, non-invasive tumors despite conservative treatments. ${ }^{1}$

Robot-assisted (RA) procedures are increasingly being performed as minimally invasive surgical approaches. Robot-assisted radical cystectomy (RARC) has the advantages of decreased blood loss, decreased time to flatus, decreased time to bowel movement, and decreased analgesic use, all of which may allow adjuvant chemotherapy administration at more appropriate time when needed and/or increased quality of life in post-surgery period compared with ORC. ${ }^{1,2}$

Many patients with ORC indication are candidates for RARC except some special contraindications such as presence of intra-abdominal adhesions preventing the placement of the ports and patients who cannot tolerate the pneumoperitoneum and/or steep Trendelenburg (ST) position. ${ }^{3}$

Additionally, body mass index $(\mathrm{BMI})>30 \mathrm{~kg} / \mathrm{m}^{2}$, presence of extravesical disease, bulky lymphadenopathy, previous vascular surgery, previous distal colorectal surgery, previous pelvic radiation, previous pelvic trauma, and/or preexisting cardiovascular/pulmonary disease that is compromised with positioning are not
Correspondence: Mevlana Derya Balbay Department of Urology, American Hospital, Guzelbahce Sok, No: 20, 34365 Istanbul, Turkey Email mderyabalbay@yahoo.com 
certainly contraindicated but unwanted characteristics. Besides, as the surgeon gains experience, RARC may also be performed successfully in the patients possessing these conditions.

\section{Obesity}

Obesity emerges as an important health care problem associated with increased health care costs, higher risk of comorbidity, and premature deaths. ${ }^{4}$ Two large series evaluated obese patients in terms of ORC outcomes. Chang et al reported that obesity leads to higher blood loss and longer operative times. ${ }^{5}$ Lee et al reported that elevated BMI results in increased complication rate, operative time, and estimated blood loss. ${ }^{6}$

Poch et al evaluated the influence of BMI on RARC and intracorporeal ileal conduit diversion. ${ }^{7}$ They found that BMI $>30 \mathrm{~kg} / \mathrm{m}^{2}$ results in significantly increased blood loss compared with normal and overweight patients - mean 500 $\mathrm{mL}$. However, that was less than reported for obese patients in large open series. BMI was not found to be associated with increased length of stay (LOS) or increased severity of complications compared to the open series. ${ }^{7}$ However, the number of complications was similar in all BMI classes in contrast with previous open series. ${ }^{7}$ Moreover, Butt et al found no difference in operative time, blood loss, and complication rates between all BMI subgroups in a 51-patient series that underwent RARC and extracorporeal ileal conduit. ${ }^{8}$

Due to the literature and to our own experience, RARC with extracorporeal or intracorporeal urinary diversion might be performed safely in overweight and obese patients.

\section{Advanced age}

Bladder cancer is most commonly seen in elderly patients with a significantly increased incidence by the age of 70 years. ${ }^{9}$

Coward et al reported the operative outcomes and pathologic results of 99 patients who underwent RARC, categorized by age (younger vs $>70$-year-old).${ }^{10}$ There was no statistically significant difference between the two groups in terms of estimated blood loss, interval to bowel movement, and interval to discharge. ${ }^{10}$ Despite the significantly higher American Society of Anesthesiology (ASA) scores observed in elderly patients compared to the younger patients, they did not find significant difference in complication rates. ${ }^{10}$ Richards et al compared ORC and RARC performances at $>75$-year-old patients. ${ }^{11}$ Estimated blood loss, median hospital stay, and number of total and major complications were significantly lower in the RARC group. ${ }^{11}$
Groote et al showed that median operative time, median estimated blood loss, median LOS, reoperation rate, and perioperative complications for low-grade and high-grade tumors in addition to overall complications according to the Clavien-Dindo system were similar between patients $<80$ years and octogenarians. ${ }^{12}$ Octogenarian patients undergoing RARC who have high risk in terms of non-muscle-invasive and muscle-invasive bladder cancer were not found to have increased risk for peri- and postoperative morbidity and mortality rates. ${ }^{12}$

RARC have several challenges due to the additional comorbidities increasing by age in elderly patients. Another concern is that elderly patients might less tolerate complications and comorbidities compared to younger patients who have more physiological capacity. Aging is accompanied by a decrease in the lung tissue elasticity and a decreased response to hypoxemia and/or hypercapnia. ${ }^{13}$ However, despite these physiologic changes, the pneumoperitoneum as well as the ST position in RARC may well be tolerated by elderly. ${ }^{11}$

\section{Cardiopulmonary performance}

Determining the cardiopulmonary performance status of patients is one of the most important pre-assessments before radical cystectomy, especially in the elderly. This evaluation contributes to predict the postoperative adverse outcomes. ${ }^{14-16}$ The tools that are mostly used for this purpose are ASA index, Charlson comorbidity index, and Eastern Cooperative Oncology Group performance index.

Cardiopulmonary exercise testing (CPET) is another method with distinct advantage of measuring the physiological oxygen exchange efficiency and cardiorespiratory function (CRF) individually. Variables derived from CPET including anaerobic threshold (AT) and oxygen consumption $\left(\mathrm{VO}_{2}\right)$ are predictors of major complications and LOS after ORC and RARC with extracorporeal urinary diversion (eRARC). Patients with a low AT $(<11 \mathrm{~mL} / \mathrm{kg} / \mathrm{min})$ appear to be at high risk of complications and death. The peak $\mathrm{VO}_{2}$ and ventilatory equivalent for carbon dioxide $\left(\mathrm{VE} / \mathrm{VCO}_{2}\right)$ are also important as they provide a measure of lung efficiency. ${ }^{17}$ This allows clinicians to create a personalized risk profile with which postoperative morbidity and mortality can be predicted. ${ }^{18-20}$

Prentis et al evaluated 82 patients undergoing ORC by CPET. They found a significant difference in LOS between the patients who had a major (Clavien score $>3$ ) perioperative complication and the patients who did not (16 vs 30 days; $P<0.001) .{ }^{18}$ Tolchard et al evaluated 105 patients in terms of the relationship between CPET and complications after RC 
undergoing ORC or eRARC and they found AT was negatively, and $\mathrm{VE} / \mathrm{VCO}_{2}$ positively, correlated with complications and LOS. ${ }^{19}$ Lamb et al evaluated 82 patients undergoing RARC with intracorporeal urinary diversion (iRARC). They found no significant difference in AT, peak $\mathrm{VO}_{2}$, or VE/ $\mathrm{VCO}_{2}$ between patients who had complications and those who did not. ${ }^{21}$ Undergoing iRARC, low cardiorespiratory reserve did not predict the occurrence of major complications and LOS.

The morbidity and mortality associated with RC have been reduced in recent years; therefore, iRARC may present as an additional means of improving patient outcomes.

\section{Preoperative anemia}

Preoperative anemia is commonly seen and the incidence increases in patients receiving neoadjuvant chemotherapy. Also hematuria and the chronic process of the disease may lead to anemia, and thus the incidence of anemia is higher in elderly patients than in younger patients. Preoperative anemia may negatively affect the perioperative outcomes.

Tan et al evaluated iRARC performed in 166 patients in a study in which 72 of them were anemic. ${ }^{22}$ Conversely, the results suggested that preoperative anemia was not associated with median LOS, 30- and 90-day morbidity, and 90-day readmission rates in iRARC-performed patients. Anemia alone was not found to be associated with higher complication rates. Besides, blood transfusion requirement was the most deterministic factor associated with perioperative complications. $^{22}$

\section{Oncologic outcomes}

In the European Association of Urology Robotic Urology Section (ERUS) Scientific Working Group database, 717 patients were identified at 9 different institutions who underwent iRARC. ${ }^{23}$ The median follow-up was 31 months. Recurrence-free survival (RFS) rates at 3, 12, and 24 months were $95.9 \%, 80.2 \%$, and $74.6 \%$, respectively. Any unusual recurrence pattern after RARC was not identified in this multi-institutional study. ${ }^{23}$ Early recurrences at any site were detected in $4.1 \%$ of patients at 3 months, $19.8 \%$ at 12 months, and $25.4 \%$ at 24 months similar with the rates in ORC series. Eighteen percent of RARC patients had positive lymph nodes and $32.6 \%$ had pT3/4 disease. Early recurrence rates and recurrence sites were similar with those for ORC series. ${ }^{24-26}$

Tan et al evaluated 184 bladder cancer patients who underwent RC. ${ }^{22}$ Number of ORC cases was 94 and the number of iRARC cases was 90. Median follow-up without recurrence was 33.8 months for ORC and 16.1 months for iRARC. Overall, median follow-up was 22.8 months. Between the two groups, there was no significant difference in cancer-specific survival (80.9\% ORC vs 84.4\% iRARC), RFS (65.9\% ORC vs $75.2 \%$ iRARC), and overall survival (OS) (72.5\% ORC vs iRARC 79.2\%) at 24 months. No significant difference was observed in age, sex, precystectomy grade, precystectomy $\mathrm{T}$ stage, and lymph node yield between ORC and iRARC. Most of the cases were urothelial cell carcinoma (UCC) and no significant difference was found in precystectomy pathological $\mathrm{T}$ stage. No significant difference was detected between number of lymph nodes resected, local and metastatic RFS, recurrence patterns, and oncological outcomes. ${ }^{27}$

Collins et al evaluated iRARC-performed 70 patients whose data were collected prospectively and reviewed retrospectively. The unadjusted survival rates at 24 months for recurrence-free, cancer-specific-free, and overall survival were $80.7 \%, 88.9 \%$, and $88.9 \%$, respectively. They found that the oncologic outcome results were similar with ORC series exhibiting iRARC as a safe and feasible method. ${ }^{28}$

\section{Concomitant surgery}

Alkan et al reported a case of simultaneous robotic nephroureterectomy (RANU), genital tract and nerve-sparing RARC with super-extended pelvic lymph node (LN) dissection, and intracorporeal Studer urinary reconstruction on a female patient with muscle-invasive bladder and a distal ureteral tumor. ${ }^{29}$ Barros et al evaluated eight patients (seven males, one female) with bladder cancer and of those, seven patients underwent laparoscopy and one patient underwent RARC with RANU (unilateral $[n=6]$, bilateral $[n=2]) \cdot{ }^{30}$ Ou et al simultaneously performed RANU and RARC on eight patients (five females, three males) with UCC and uremia. ${ }^{31}$ Justin et al reported a patient who underwent RA laparoscopic combined nephroureterectomy and radical cystoprostatectomy with extended PLND and extracorporeal ileal conduit urinary diversion. ${ }^{32}$

All of the studies concluded that simultaneous RANU and RARC may be safely performed. En bloc removal of kidney, ureter, prostate, and bladder may be done safely by utilizing minimally invasive techniques such as robotic surgery that seems to be technically safe and feasible with excellent oncological outcomes and minimal complications.

\section{Patient positioning (steep Trendelenburg position) and pneumoperitoneum}

$\mathrm{RARC}$ is performed in $30^{\circ} \mathrm{ST}$ position. This may adversely affect intracranial pressure (ICP), eye and middle ear pressures during prolonged operative time periods.

Positioning patient in the ST position in combination with pneumoperitoneum leads to cerebral oxygen saturation 
decrease by a clinically insignificant value of $<5 \%$ over a time period exceeding $4 \mathrm{~h} .{ }^{33} \mathrm{ICP}$ increases in the ST position and additional pneumoperitoneum can further elevate ICP. Increased ICP results in reduced venous drainage of the brain leading to cerebral edema. Increased ICP and cerebral edema may result in cerebral hypoperfusion and ischemia. ${ }^{34}$ Pandey et al reported two patients who underwent a prolonged ST positioning of about 7-10 h for RARC. The patients developed cerebral edema with consequent neurologic damage. ${ }^{35}$

Severe neurological complications are generally rare. Minor clinical symptoms such as nausea and headache related with increased ICP are likely to be seen more frequently after laparoscopy. ${ }^{36-38}$

The increase in ICP may lead to partial or complete vision loss, retinal detachment, and ischemic optic neuropathy. ${ }^{39-41}$ Intraocular pressure (IOP) increases significantly in the ST position. ${ }^{42}$ This increase results in hemodynamic changes in eye. In RARC, ocular complication risk is low despite the requirement to stay in the ST position for a long time. Ozcan et al evaluated 53 patients (robot assisted laparoscopic radical prostatectomy: 43; iRARC: 10) intraoperatively in terms of the effect of the ST on IOP, resistive index of the central retinal artery, and venous impedance index of the central retinal vein prosectively. ${ }^{43}$ There was no difference between the IOP values of the right and left eyes in both groups. The highest IOP values were identified in patients under anesthesia during intraperitoneal $\mathrm{CO}_{2}$ insufflation or at the end of the operation. Despite staying in the ST for a long time, ophthalmologic examination was normal. ${ }^{43}$

Routine preoperative ophthalmologic examination is not warranted and patients should be questioned before the operation with regard to any history of ophthalmic disease such as glaucoma. Ocular hypertension and/or glaucoma may be a risk factor in RARC. The IOP values may also affect glaucoma patients who possess ocular blood flow abnormalities. Elevated IOP (up to $45 \mathrm{mmHg}$ ) and short-term changes in ocular perfusion pressure do not affect normal eyes due to autoregulatory mechanism on blood flow to the optic nerve head. ${ }^{44}$ It is proposed that patients with ocular preexisting diseases such as glaucoma should be referred to an ophthalmologist preoperatively.

Bozkirli et al evaluated ASA physical status I-III in 35 patients, aged 18-80, undergoing robot-assisted radical prostatectomy (RARP) in the ST position prospectively to evaluate middle ear pressure (MEP) changes related to the ST position and $\mathrm{CO}_{2}$ pneumoperitoneum. ${ }^{45}$ They found that the ST position with pneumoperitoneum in RARC significantly increases MEP and this increase may continue through the first hour postoperation. The prolonged response may be related with the $\mathrm{CO}_{2}$ diffusion to the middle ear or the time and degree of the ST position. ${ }^{45}$

Increase in MEP may lead to undesirable clinical outcomes. Postoperative intractable nausea, vomiting, rupture of membranes, and even facial nerve damages may be seen due to significant and long-term increases in MEP. ${ }^{46-48}$ During the preanesthetic evaluation of patients, detailed anamnesis of ear diseases should be questioned and consultation by an ear, nose, and throat specialist should be done before RARP and RARC surgeries.

There is no sufficient evidence about the safety of the ST position while performing RARC in the presence of cerebral aneurysm, arteriovenous malformation, and meningioma. As a matter of fact, many patients do not know that they have such a special condition. Therefore, further studies are needed on this issue.

\section{Unexpected conditions}

We previously reported two cases of RARC with bilateral extended PLND and intracorporeal Studer pouch formation in patients with duplicated right ureters. ${ }^{49}$ Ureteric duplication is a rarely seen malformation of the urinary tract more commonly seen in females. Duplicated ureters might be underdiagnosed on CT and we do not think that this rare entity is a contraindication to use the robot in this complicated procedure. Ureteric duplication can be successfully managed at RARC. ${ }^{49}$

In one of our previous experiences, when we opened the ileum in RARC to form a Studer pouch intracorporeally, we saw the moving parasites on the mucosal surface. They were 5-mm sized and yellow-white colored. We removed all of them with the laparoscopic grasper and sent them for pathologic and parasitologic evaluation. Then we completed iRARC successfully. The pathologic and parasitologic evaluation results were reported as Taenia saginata. So, stool sample evaluation should be done in the preoperative period for the possible presence of intestinal parasitic diseases particularly in patients with bladder cancer coming from areas with an increased incidence of intestinal parasitic diseases before opening the bowel segments during surgery to perform radical cystectomy and urinary diversion. ${ }^{50}$

\section{Complications of lengthy surgery, pneumoperitoneum, lithotomy position, compartment syndrome}

One of the disadvantages of robotic approach is the longer operation time that was reported by a very recent 
systematic review and meta-analysis. ${ }^{51}$ Longer operation time is expected to lead to longer exposure to pneumoperitoneum that might particularly have a negative impact on the lung function that is explained above. Yamada et al stated that lower extremity neuropathy is a well-recognized complication that arises during robotic surgeries in lithotomyTrendelenburg position due to the persistent compression to the nerves, and robotic prostate cancer and bladder cancer surgeries are examples. ${ }^{52}$

Lower limb compartment syndrome was reported to occur in RARP that has a low incidence. Long operation times, surgical inexperience, poor patient positioning, obesity, and vascular disease were suggested as risk factors for this condition. ${ }^{53,54}$

\section{Conclusion}

iRARC is increasingly being performed with a remarkable progressing success. Except the special contraindications, the technique has been suggested to be safe, reproducible, and oncologically feasible. Increasing expertise of the surgeon and technological improvements are expected to provide better operative and functional outcomes.

\section{Disclosure}

The authors report no conflicts of interest in this work.

\section{References}

1. Herr HW, Dotan Z, Donat SM, Bajorin DF. Defining optimal therapy for muscle invasive bladder cancer. J Urol. 2007;177:437-443.

2. Collins JW, Wiklund NP. Totally intracorporeal robot-assisted radical cystectomy: optimizing total outcomes. BJU Int. 2014;114(3):326-333.

3. Chan KG, Guru KA, Wiklund P, et al; Pasadena Consensus Panel. Robotassisted radical cystectomy and urinary diversion: technical recommendations from the Pasadena Consensus Panel. Eur Urol. 2015;67(3): 423-431.

4. Pi-Sunyer FX. National Heart, Lung and Blood Institute. Clinical Guideline On The Identification, Evaluation, And Treatment Of Overweight And Obesity In Adults: The Evidence Report. Bethesda, MD: US Department of Health and Human Services, National Institutes of Health, National Heart, Lung and Blood Institute; 1998.

5. Chang SS, Jacobs B, Wells N, Smith JA Jr, Cookson MS. Increased body mass index predicts increased blood loss during radical cystectomy. $J$ Urol. 2004;171(3):1077-1079.

6. Lee CT, Dunn RL, Chen BT, Joshi DP, Sheffield J, Montie JE. Impact of body mass index on radical cystectomy. J Urol. 2004;172(4 Pt 1): 1281-1285.

7. Poch MA, Stegemann A, Chandrasekhar R, Hayn M, Wilding G, Guru KA. Does body mass index impact the performance of robot-assisted intracorporeal ileal conduit? J Endourol. 2012;26(7):857-860.

8. Butt ZM, Perlmutter AE, Piacente PM, et al. Impact of body mass index on robot-assisted radical cystectomy. JSLS. 2008;12(3):241-245.

9. American Cancer Society. Probability of developing invasive cancers over selected age intervals by sex, US 2002-2004. American Cancer Society. Available from: www.cancer.org/downloads/stt/CFF2008Table_pg14.pdf. Accessed October 24, 2009.
10. Coward RM, Smith A, Raynor M, Nielsen M, Wallen EM, Pruthi RS. Feasibility and outcomes of robot-assisted laparoscopic radical cystectomy for bladder cancer in older patients. Urology. 2011;77(5): 1111-1114.

11. Richards KA, Kader AK, Otto R, Pettus JA, Smith JJ 3rd, Hemal AK. Is robot-assisted radical cystectomy justified in the elderly? A comparison of robotic versus open radical cystectomy for bladder cancer in elderly $>75$ years old. $J$ Endourol. 2012;26(10):1301-1306.

12. De Groote R, Gandaglia G, Geurts N, et al. Robot-assisted radical cystectomy for bladder cancer in octogenarians. $J$ Endourol. 2016;30(7):792-798.

13. Shariat SF, Milowsky M, Droller MJ. Bladder cancer in the elderly. Urol Oncol. 2009;27(6):653-667.

14. Boorjian SA, Kim SP, Tollefson MK, et al. Comparative performance of comorbidity indices for estimating perioperative and 5-year all cause mortality following radical cystectomy for bladder cancer. $J$ Urol. 2013;190(1):55-60.

15. Charlson ME, Pompei P, Ales KL, MacKenzie CR. A new method of classifying prognostic comorbidity in longitudinal studies: development and validation. J Chronic Dis. 1987;40(5):373-383.

16. Royal College of Surgeons and the Department of Health. The higher risk general surgical patient: towards improved care for a forgotten group. London; 2011. Available from: http://www.rcseng.ac.uk/ publications/docs/higher-risk-surgical-patient/@@download/pdffile/ higher_risk_surgical_patient_2011_web.pdf. Accessed October 5, 2017.

17. Wasserman K, Hansen J, Sue D, Stringer W, Whipp B. Principles of Exercise Testing and Interpretation. 4th ed. Philadelphia, PA: Lippincott Williams and Wilkins; 2004.

18. Prentis JM, Trenell MI, Vasdev N, et al. Impaired cardiopulmonary reserve in an elderly population is related to postoperative morbidity and length of hospital stay after radical cystectomy. BJU Int. 2013;112(2): E13-E19.

19. Tolchard S, Angell J, Pyke M, et al. Cardiopulmonary reserve as determined by cardiopulmonary exercise testing correlates with length of stay and predicts complications after radical cystectomy. BJUInt. 2015;115(4): 554-561.

20. Hightower CE, Riedel BJ, Feig BW, et al. A pilot study evaluating predictors of post-operative outcome after major abdominal surgery: physiological capacity compared with the ASA physical status classification system. Br J Anaesth. 2010;104(4):465-471.

21. Lamb BW, Tan WS, Eneje P, et al. Benefits of robotic cystectomy with intracorporeal diversion for patients with low cardiorespiratory fitness: a prospective cohort study. Urol Oncol. 2016;34(9):417.e17-e23.

22. Tan WS, Lamb BW, Khetrapal P, et al. Blood transfusion requirement and not preoperative anemia are associated with perioperative complications following intracorporeal robot-assisted radical cystectomy. J Endourol. 2017;31(2):141-148.

23. Collins JW, Hosseini A, Adding C, et al. Early recurrence patterns following totally intracorporeal robot-assisted radical cystectomy: results from the EAU Robotic Urology Section (ERUS) Scientific Working Group. Eur Urol. 2017;71(5):723-726.

24. Sonpavde G, Khan MM, Lerner SP, et al. Disease-free survival at 2 Or 3 years correlates with 5-year overall survival of patients undergoing radical cystectomy for muscle invasive bladder cancer. J Urol. 2011;185(2): 456-461.

25. Wallmeroth A, Wagner U, Moch H, Gasser TC, Sauter G, Mihatsch MJ. Patterns of metastasis in muscle-invasive bladder cancer (Pt2-4): an autopsy study of 367 patients. Urol Int. 1999;62:69-75.

26. Kim B, Choi HJ, Kim MH, Cho KS. Recurrence patterns of bladder transitional cell carcinoma after radical cystectomy. Acta Radiol. 2012;53(8):943-949.

27. Tan WS, Sridhar A, Ellis G, et al. Analysis of open and intracorporeal robotic assisted radical cystectomy shows no significant difference in recurrence patterns and oncological outcomes. Urol Oncol. 2016;34(6): 257.e1-e9. 
28. Tyritzis SI, Hosseini A, Collins J, et al. Oncologic, functional, and complications outcomes of robot-assisted radical cystectomy with totally intracorporeal neobladder diversion. Eur Urol. 2013;64(5):734-741.

29. Alkan E, Canda AE, Turan M, Balbay MD. Simultaneous robot assisted laparoscopic radical nephroureterectomy; genital tract and paravaginal nerve sparing radical cystectomy; superextended lymph node dissection and intracorporeal studer pouch reconstruction for bladder cancer: robotic hat-trick. Cent European J Urol. 2014;67(3):257-260.

30. Barros R, Frota R, Stein RJ, Turna B, Gill IS, Desai MM. Simultaneous laparoscopic nephroureterectomy and cystectomy: a preliminary report. Int Braz J Urol. 2008;34(4):413-421.

31. Ou YC, Yang CR, Yang CK, Cheng CL, Hemal AK. Simultaneous robotassisted nephroureterectomy and cystectomy in patients with uremia and multifocal urothelial carcinoma. J Endourol. 2011;25(6):979-984.

32. Benabdallah JO, Hampton LJ, Guruli G, Grob BM. Robot-assisted laparoscopic combined nephroureterectomy and cystoprostatectomy: an initial report and review of the literature. J Robot Surg. 2012;6(2): 159-162.

33. Closhen D, Treiber AH, Berres M, et al. Robotic assisted prostatic surgery in the trendelenburg position does not impair cerebral oxygenation measured using two different monitors: a clinical observational study. Eur J Anaesthesiol. 2014;31(2):104-109.

34. Awad H, Walker CM, Shaikh M, Dimitrova GT, Abaza R, O’Hara J. Anesthetic considerations for robotic prostatectomy: a review of the literature. J Clin Anesth. 2012;24(6):494-504.

35. Pandey R, Garg R, Darlong V, Punj J, Chandralekha, Kumar A. Unpredicted neurological complications after robotic laparoscopic radical cystectomy and ileal conduit formation in steep trendelenburg position: two case reports. Acta Anaesthesiol Belg. 2010;61(3):163-166.

36. Danic MJ, Chow M, Alexander G, Bhandari A, Menon M, Brown M. Anesthesia considerations for robotic-assisted laparoscopic prostatectomy: a review of 1,500 cases. J Robot Surg. 2007;1(2):119-123.

37. Ficarra V, Novara G, Rosen RC, et al. Retropubic, laparoscopic, and robot-assisted radical prostatectomy: a systematic review and cumulative analysis of comparative studies. Eur Urol. 2012;62(3):405-417.

38. Cooke SJ, Paterson-Brown S. Association between laparoscopic abdominal surgery and postoperative symptoms of raised intracranial pressure. Surg Endosc. 2001;15(7):723-725.

39. Weber ED, Colyer MH, Lesser RL, Subramanian PS. Posterior ischemic optic neuropathy after minimally invasive prostatectomy. J Neuroophthalmol. 2007;27(4):285-287.

40. Martin JT. The trendelenburg position: a review of current slants about head down tilt. AANA J. 1995;63(1):29-36.

41. Henny CP, Hofland J. Laparoscopic surgery: pitfalls due to anesthesia, positioning, and pneumoperitoneum. Surg Endosc. 2005;19(9):1163-1171.
42. Hewer CL. The physiology and complications of the trendelenburg position. Can Med Assoc J. 1956;74(4):285-288.

43. Ozcan MF, Akbulut Z, Gurdal C, et al. Does steep trendelenburg positioning effect the ocular hemodynamics and intraocular pressure in patients undergoing robotic cystectomy and robotic prostatectomy? Int Urol Nephrol. 2017;49(1):55-60.

44. Pillunat LE, Anderson DR, Knighton RW, Joos KM, Feuer WJ. Autoregulation of human optic nerve head circulation in response to increased intraocular pressure. Exp Eye Res. 1997;64(5):737-744.

45. Bozkirli F, Bedirli N, Akçabay M. Effects of steep Trendelenburg position and pneumoperitoneum on middle ear pressure in patients undergoing robotic radical prostatectomy. Turk J Med Sci. 2017;47(1): 295-299.

46. Nader ND, Simpson G, Reedy RL. Middle ear pressure changes a er nitrous oxide anesthesia and its effect on postoperative nausea and vomiting. Laryngoscope. 2004;114(5):883-886.

47. Perreault L, Normandin N, Plamondon L, et al. Tympanic membrane rupture after anesthesia with nitrous oxide. Anesthesiology. 1982;57(4): 325-326.

48. Garcia Callejo FJ, Velert Vila MM. [Facial paralysis after non-otologic surgery under general anesthesia]. Acta Otorrinolaringol Esp. 1998;49(2):173-175. Spanish.

49. Canda AE, Dogan B, Atmaca AF, Akbulut Z, Balbay MD. Ureteric duplication is not a contraindication for robot-assisted laparoscopic radical cystoprostatectomy and intracorporeal Studer pouch formation. JSLS. 2011;15(4):575-579.

50. Canda AE, Asil E, Balbay MD. An unexpected resident in the ileum detected during robot-assisted laparoscopic radical cystoprostatectomy and intracorporeal Studer pouch formation: Taenia saginata parasite. $J$ Endourol. 2011;25(2):301-303.

51. Son SK, Lee NR, Kang SH, Lee SH. Safety and effectiveness of robotassisted versus open radical cystectomy for bladder cancer: a systematic review and meta-analysis. J Laparoendosc Adv Surg Tech A. Epub 2017 Mar 28.

52. Yamada Y, Fujimura T, Fukuhara H, et al. Measuring contact pressure of lower extremities in patients undergoing robot-assisted radical prostatectomy. Urol Int. 2016;96(3):268-273.

53. Song JH, Kaplan JR, Abbott D, Gewirtz E, Hauck E, Eun DD. Obturator compartment syndrome secondary to pelvic hematoma after robot-assisted laparoscopic radical prostatectomy. J Endourol Case Rep. 2016;2(1): 141-143.

54. Pridgeon S, Bishop CV, Adshead J. Lower limb compartment syndrome as a complication of robot-assisted radical prostatectomy: the UK experience. BJU Int. 2013;112(4):485-488.
Robotic Surgery: Research and Reviews

\section{Publish your work in this journal}

Robotic Surgery: Research and Reviews is an international, peer reviewed, open access, online journal publishing original research, commentaries, reports, and reviews on the theory, use and application of robotics in surgical interventions. Articles on the use of supervisory-controlled robotic systems, telesurgical devices, and shared-control systems are

\section{Dovepress}

invited. The manuscript management system is completely online and includes a very quick and fair peer review system, which is all easy to use. Visit http://www.dovepress.com/testimonials.php to read real quotes from published authors. 